\title{
Configuration of a multielement interferometer covering hexagonal domains in the spatial-frequency plane
}

\author{
L. E. Kopilovich^ \\ Institute of Radio Physics and Electronics, Kharkov 61085, Ukraine \\ Received 25 July 2001 / Accepted 27 September 2001

\begin{abstract}
A class of threefold symmetric configurations of an interferometer completely covering a hexagonal domain of a given size in the spatial-frequency plane and thus ensuring practically equal resolution in all beam cross-sections is described. The configurations are compactly arranged and have a minimized number of elements.
\end{abstract}

Key words. instrumentation: interferometers

\section{Introduction}

In recent times, building of large radio telescopes became urgent. Constructions of multielement 2-D interferometers which met various optimization criteria were suggested by Keto (1997), Lonsdale \& Cappallo (1999), and Kogan (2000). Among the admissible configurations, those covering a hexagonal domain in the spatial-frequency plane ( $u, v$-plane) are also of interest (Sodin \& Kopilovich 2000, 2001 - further, SK). Such a coverage can be made complete and uniform, i.e. without holes, that is an important advantage.

The development of a method for constructing optimized configurations which cover completely a hexagonal $u, v$-domain of a given size is now required. The optimization may consist of minimizing the area of the element arrangement or their number. Such configurations placed on a minimum area were first suggested by Haurbich (1968) who arranged elements in triangles in a hexagonal grid. If one fills up the perimeter of an equilateral triangle having sidelength $R+1$ with elements, then the hexagonal $u, v$-domain of radius $R$ would be covered (the radius of a regular hexagon is the distance between its centre and vertex). Clearly, the number of elements needed in this case is very redundant. Another simple solution of the problem is to fill up five sides of a regular hexagon of a given radius by elements which provide complete coverage of a hexagonal domain of the double radius in the $u, v$-plane; however, the number of elements in such a construction, though smaller than that required to cover the same $u, v$-domain as in the previous case $(5 R+1$ against $6 R$ ), is also a surplus. The problem of constructing the most compact configurations with a minimized number of elements on hexagonal grids has been studied by

\footnotetext{
* e-mail: kopil@ire.kharkov.ua
}

Kopilovich (1999); some examples of these configurations are given in SK.

To further reduce the number of elements, one is led to make their compactness requirements less stringent. However,we shall restrict the area of the element arrangement so that it will not be as large as in the case of nonredundant configurations (Golay 1971).

Here, we suggest a way to obtain configurations with a minimized number of elements arranged in a hexagonal grid $H_{r}$ of radius $r<R$, where $R$ is the radius of the hexagonal $u, v$-domain $H_{R}$ to be covered. The proposed approach enables one to describe a class of interferometer configurations covering hexagonal domains of any radius in the $u, v$-plane.

\section{The building of configurations}

The essential condition which makes it possible to realize our approach is that we consider only configurations possessing threefold symmetry, i.e. invariant to rotation by $120^{\circ}$ around a certain centre of symmetry. There are two types of such constructions in hexagonal grids. For those of type I the symmetry centre coincides with that of the hexagon as shown in Figs. 1a,b. The grids in the figures are divided into three sectors (excluding the central node), and the oblique coordinates having their origin in the hexagon centre are introduced. One can see that among the coordinates of a certain configuration element $\left(x_{1}, y_{1}\right)$ in sector 1 and of the corresponding elements $\left(x_{2}, y_{2}\right)$ and $\left(x_{3}, y_{3}\right)$ in sectors 2 and 3 , respectively, the following relations hold

$x_{2}=-\left(x_{1}+y_{1}\right), y_{2}=x_{1} ; \quad x_{3}=y_{1}, y_{3}=x_{2}$. 

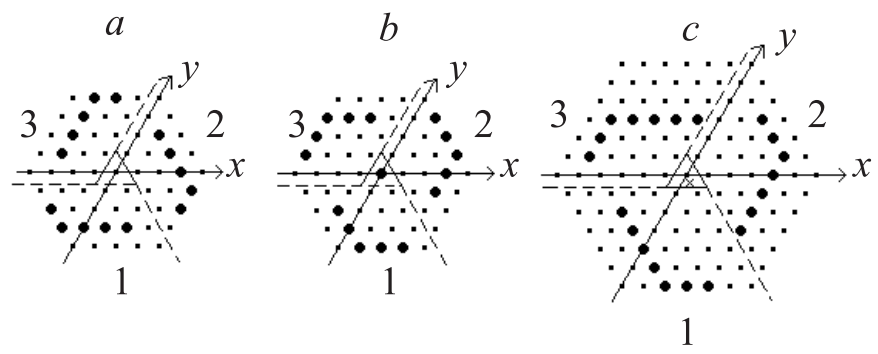

Fig. 1. Examples of configurations with a threefold symmetry in a hexagonal grid: a) the symmetry centre of the configuration coincides with the hexagon centre (the central element is absent); b) the same, with the central element; c) the symmetry centre (denoted with a cross) is displaced. The configuration elements are denoted with bold dots. The sectors of the grid are singled out by dotted lines.

Table 1. Characteristics of the minimum-element TCCs grouped in nine sequences: $R$, the radius of coverage; $r$, the radius of the element arrangement; $m$, the number of elements; $\left(x_{0}, y_{0}\right)$, the coordinates of the corner element in sector $1 ; L$, the length of the "corner" horizontal side in this sector; $k$ is the number of the link in a configuration sequence $(k=1,2, \ldots)$.

\begin{tabular}{ccccccc}
\hline No. & $R$ & $r$ & $m$ & $L$ & $x_{0}, y_{0}$ & type \\
\hline 1 & $12 k-8$ & $7 k-4$ & $27 k-18$ & $3 k-1$ & $2 k-1,-r$ & II \\
2 & $12 k-7$ & $7 k-4$ & $27 k-15$ & $3 k$ & $2 k-1,-r$ & I \\
3 & $12 k-6$ & $7 k-3$ & $27 k-12$ & $3 k-1$ & $2 k-1,-r$ & I \\
4 & $12 k-4$ & $7 k-2$ & $27 k-9$ & $6 k-2$ & $-2 k+1,-r+2 k-1$ & II \\
5 & $12 k-3$ & $7 k-1$ & $27 k-6$ & $3 k+1$ & $2 k,-r$ & II \\
6 & $12 k-2$ & $7 k-1$ & $27 k-3$ & $3 k+2$ & $2 k,-r$ & I \\
7 & $12 k$ & $7 k$ & $27 k$ & $3 k+1$ & $2 k,-r$ & I \\
8 & $12 k+1$ & $7 k+1$ & $27 k+3$ & $6 k$ & $-2 k,-r+2 k$ & II \\
9 & $12 k+2$ & $7 k+2$ & $27 k+6$ & $3 k+3$ & $2 k+1,-r$ & II \\
\hline
\end{tabular}

The symmetry centre of a configuration of type II is displaced relative to the hexagon centre (see Fig. 1c). In this case, Eq. (1) are transformed to

$x_{2}=-\left(x_{1}+y_{1}\right), y_{2}=x_{1}-1 ; \quad x_{3}=y_{1}+1, y_{3}=x_{2}-1$.

Note that a configuration of type II does not contain a central element.

Our approach to obtain the configurations that possess the required properties was as follows.

First, given radii of coverage from $R=4$ to 13 , we looked through threefold symmetric arrays arranged in hexagons $H_{r}$, of radius $r<R$, and found all those covering the $u, v$-domain $H_{R}$ with a minimum number of elements. At this point, it was discovered that for each value of $R$ among the obtained configurations there are those having one of the forms represented in Fig. 1. We shall name them three-cornered configurations (TCCs).

The next stage was a search for minimum-element TCCs covering hexagonal $u, v$-domains of larger radii. Obviously, a TCC is fully defined by its corner element in sector 1 and the length of the horizontal side of the "corner" (and by the presence or absence of central element). We have looked through the TCCs covering the

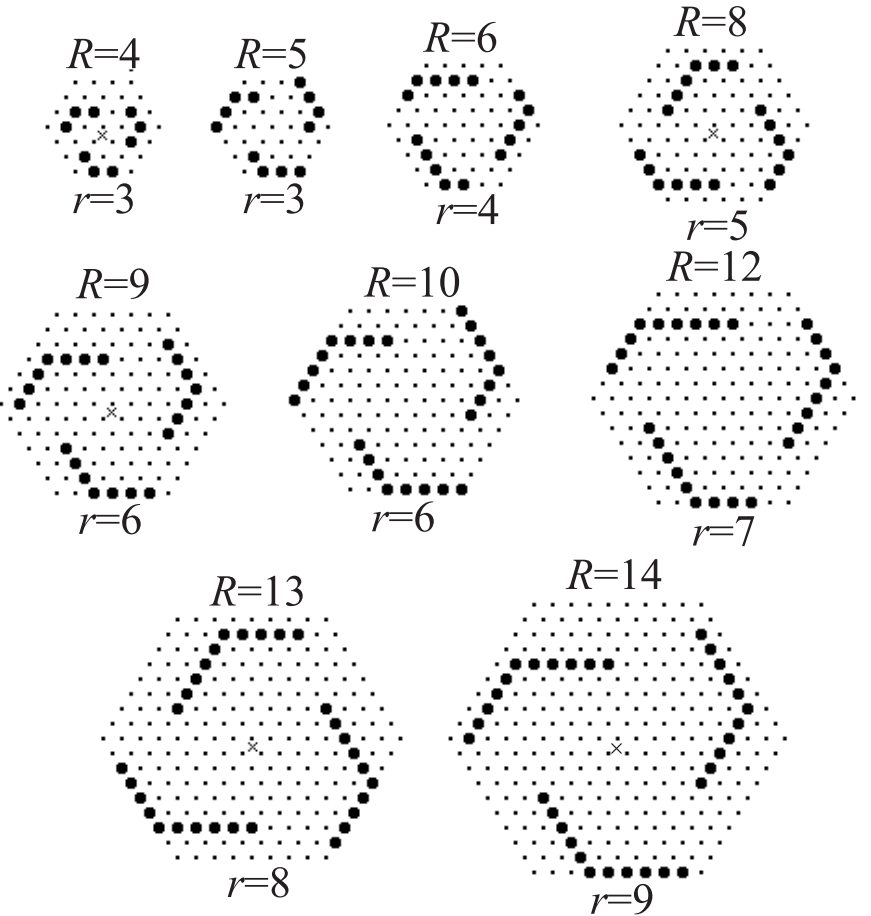

Fig. 2. The first links of the configuration sequences described in Table 1: $r$ is the radius of the element arrangement, $R$, the coverage radius, $m$ the number of elements.

$u, v$-domains of radii up to $R=45$. There exist, as a rule, a few minimum-element TCCs for each $R$, and by analyzing the whole set of these we have found pairs or triplets of a similar structure. When building, in these cases, by analogy, new TCCs on hexagons of larger radii, one can obtain nine sequences of TCCs, whose characteristics are given in Table 1. The first links of the sequences are pictured in Fig. 2. One can check for any $k$ (which is the number of a link in the sequence) that such a TCC completely covers the $u, v$-domain of the corresponding radius $R$; we have checked each sequence for $k$ up to 8 .

Note that sequences 1,5 , and 9 of Table 1 show similarities to one another, and this can also be said about sequences 2 and 6, 3 and 7, 4 and 8 . However, one can also see differences between them, so that one cannot lessen the number of the sequences while keeping the minimum number of elements in the configurations covering hexagonal $u, v$-domains of the given radii. One also sees that there are no sequences for $R=12 k-5,12 k-1$, and $12 k+3$ (i.e for $R=4 k+3)$ in the Table. The fact is that the number of elements in minimum-element TCCs for $R=4 k+3$ and $4(k+1)$ is the same, so it makes no sense to give the former one in the Table (the only exclusion is the 16-element TCC for $R=7$, which is shown in Fig. 1b; note that this is the only minimum-element configuration found having a central element). 
Table 2. The efficiency factor $\alpha$, for the TCCs from Table 1, with the number of elements up to 100 .

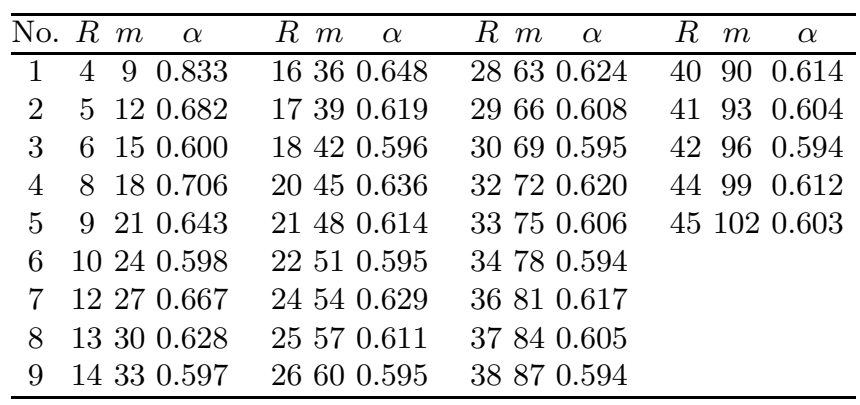

One can also see from Table 1 that for each sequence the following relationships among the characteristics $(R, r, m)$ and $\left(R_{1}, r_{1}, m_{1}\right)$ of two successive TCCs hold

$R_{1}=R+12, \quad r_{1}=r+7, \quad m_{1}=m+27$.

At large values of $R$, the ratio $r / R$ is nearly $7 / 12$, which is close to its minimum value of 0.5 .

Using the data of Table 1, one can find the dependence of $m$ on $R$ for all the TCCs obtained:

$m=2.25 R+Z$,

where $Z$ takes one of values $0,0.75$ or 1.5 , with which $m$ is an integer.

\section{The configuration efficiency}

To characterize the configurations found, one can use the efficiency factor defined as in SK:

$\alpha=N / N_{\text {id }}$,

where $N_{\mathrm{id}}$ is the number of spacings in the $u, v$-domain of the maximum radius covered completely, and $N_{\mathrm{id}}=$ $m(m-1) / 2$ is the maximum number of baselines which can be formed by m elements (for a nonredundant system). Since the number of spacings in $H_{R}$ equals $1.5 R(R+1)$, the efficiency factor for a $m$-element configuration covering such a domain is

$\alpha=3 R(R+1) /[m(m-1)]$.

The values of $\alpha$ for the TCCs from Table 1, with the number of elements up to 100, are given in Table 2. One sees
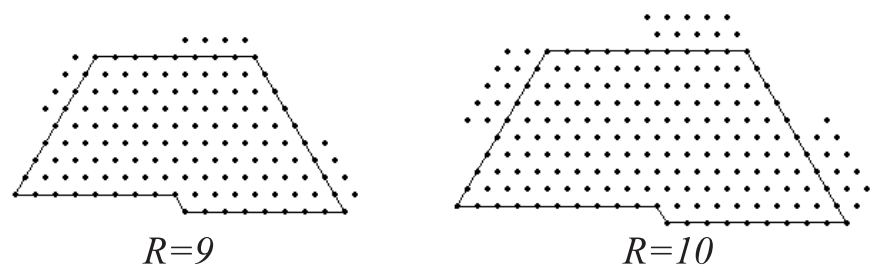

Fig. 3. Two examples of the $u, v$-plane (filled with dots) for the configurations of Fig. 2 (half the $u, v$-plane is given). The hexagonal domains covered completely are singled out.

that for each TCC sequence $\alpha$ is a decreasing function of $k$, which tends to the common limit 0.593 with $R \rightarrow \infty$.

Note that some of the obtained TCCs cover also the comparatively small regions outside the $u, v$-domain $H_{R}$ (see Fig. 3); these regions can be easily determined in each concrete case.

\section{Conclusion}

To summarize, a class of threefold symmetric configurations completely covering hexagonal domains of a given radii $R$ in the $u, v$-plane while being arranged in hexagonal domains of smaller radii $r(r / R \approx 7 / 12)$ is obtained. For small values of $R$, these configurations are the minimumelement ones among those satisfying the above requirements, and it appears that for large $R$ 's the number of their elements will also be close enough to the minimum.

\section{References}

Golay, M. E. 1971, JOSA, 61(2), 272

Haurbich, R. A. 1968, Bull. Sesmol. Soc. Amer., 58(30), 977

Keto, E. 1997, ApJ, 475, 843

Kogan, L. 2000, IEEE Trans. Antennas and Propag., 48(7), 1075

Kopilovich, L. E. 1999, Kinematics \& Physics of Celestial Bodies, 15(4), 286

Lonsdale, C. J., \& Cappalo, R. J. 1999, in Perspectives on Radio Astronomy: Technologies for Large Antenna Arrays, ed. A. B. Smolders, \& M. P. Van Harlem (Netherlands Foundation for Research in Astronomy), 243

Sodin, L. G., \& Kopilovich, L. E. 2000, Radiophys. Quant. Electron., 43(4), 345 (SK)

Sodin, L. G., \& Kopilovich, L. E. 2001, A\&A, 368, 747 (SK) 